\title{
Ion Structure Transition Enhances Charging Dynamics in Subnanometer Pores
}

\author{
Tangming Mo, ${ }^{1,2}$ Sheng Bi, ${ }^{1,2}$ Yuan Zhang, ${ }^{3,4}$ Volker Presser, ${ }^{3,4}$ \\ Xuehang Wang, ${ }^{5}$ Yury Gogotsi, ${ }^{5}$ and Guang Feng ${ }^{1,2, *}$
}

\footnotetext{
${ }^{1}$ State Key Laboratory of Coal Combustion, School of Energy and Power Engineering, Huazhong University of Science and Technology (HUST), Wuhan 430074, China

${ }^{2}$ Nano Interface Centre for Energy, School of Energy and Power Engineering, Huazhong University of Science and Technology, 430074, China

${ }^{3}$ INM - Leibniz Institute for New Materials, Campus D2 2, 66123 Saarbrücken, Germany

${ }^{4}$ Department of Materials Science and Engineering, Saarland University, 66123 Saarbrücken, Germany

${ }^{5}$ Department of Materials Science and Engineering, A. J. Drexel Nanomaterials Institute, Drexel University, Philadelphia, PA 19104, USA

*Corresponding author's email: gfeng@ hust.edu.cn (G.F.)
}

\section{Contents}

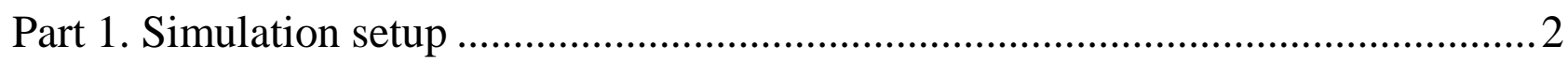

Part 2. Charging process for different pore sizes ...................................................... 3

Part 3. The capacitive behavior for different pore sizes ............................................. 4

Part 4. In-pore ion exchange during the charging process .........................................5

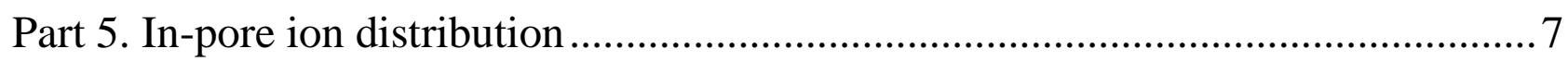

Part 6. Ion diffusion in pores of different size .......................................................... 14

Part 7. Charging dynamics of nanopore larger than $1 \mathrm{~nm}$......................................... 15 


\section{Part 1. Simulation setup}

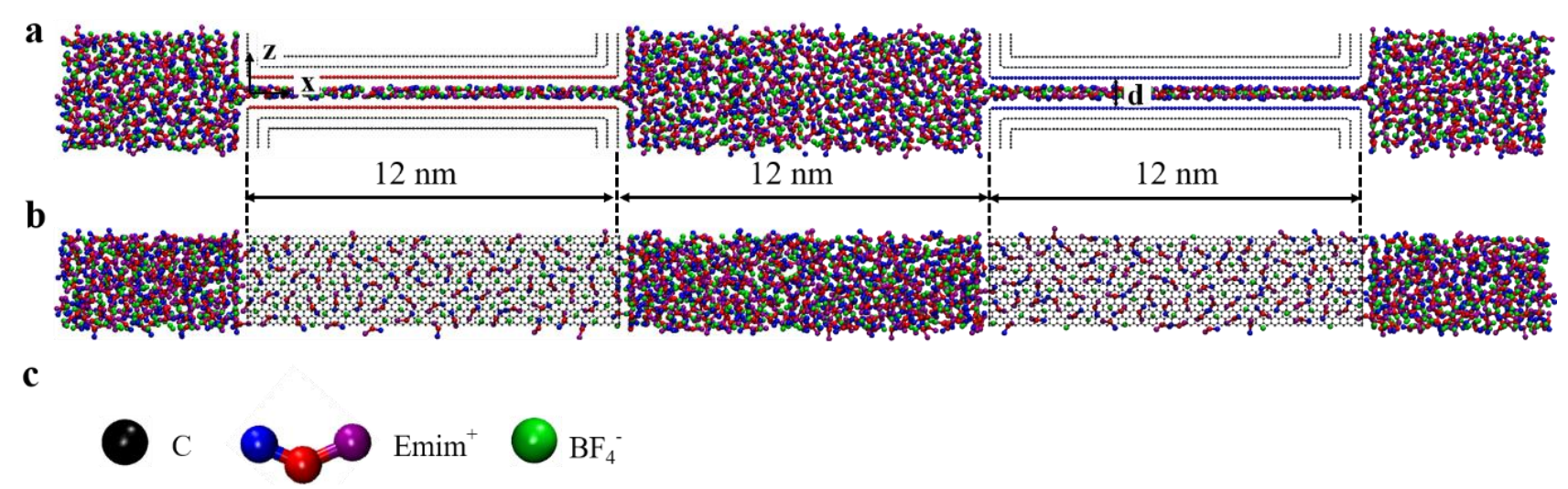

Figure S1 | Schematics of molecular dynamics simulation system setup. a-b, Front view (a) and top view (b) of MD system with two $0.75 \mathrm{~nm}$ pores. The coarse-grained model was adopted for RTIL $[\mathrm{EMIM}]\left[\mathrm{BF}_{4}\right]$. The lengths of pore and ionic liquid reservoir are both $12 \mathrm{~nm}$. The black spheres represent carbon atoms, and the colored spheres represent $[\mathrm{EMIM}]\left[\mathrm{BF}_{4}\right]$. A constant potential drop is applied on the red and blue labeled carbon atoms (the red represents positive electrode and the blue represents the negative electrode). $Z=0$ represents the position of the central plane between two pore walls. c, Molecular structure of carbon atoms and coarse-grained model of ions used in this work. 


\section{Part 2. Charging process for different pore sizes}
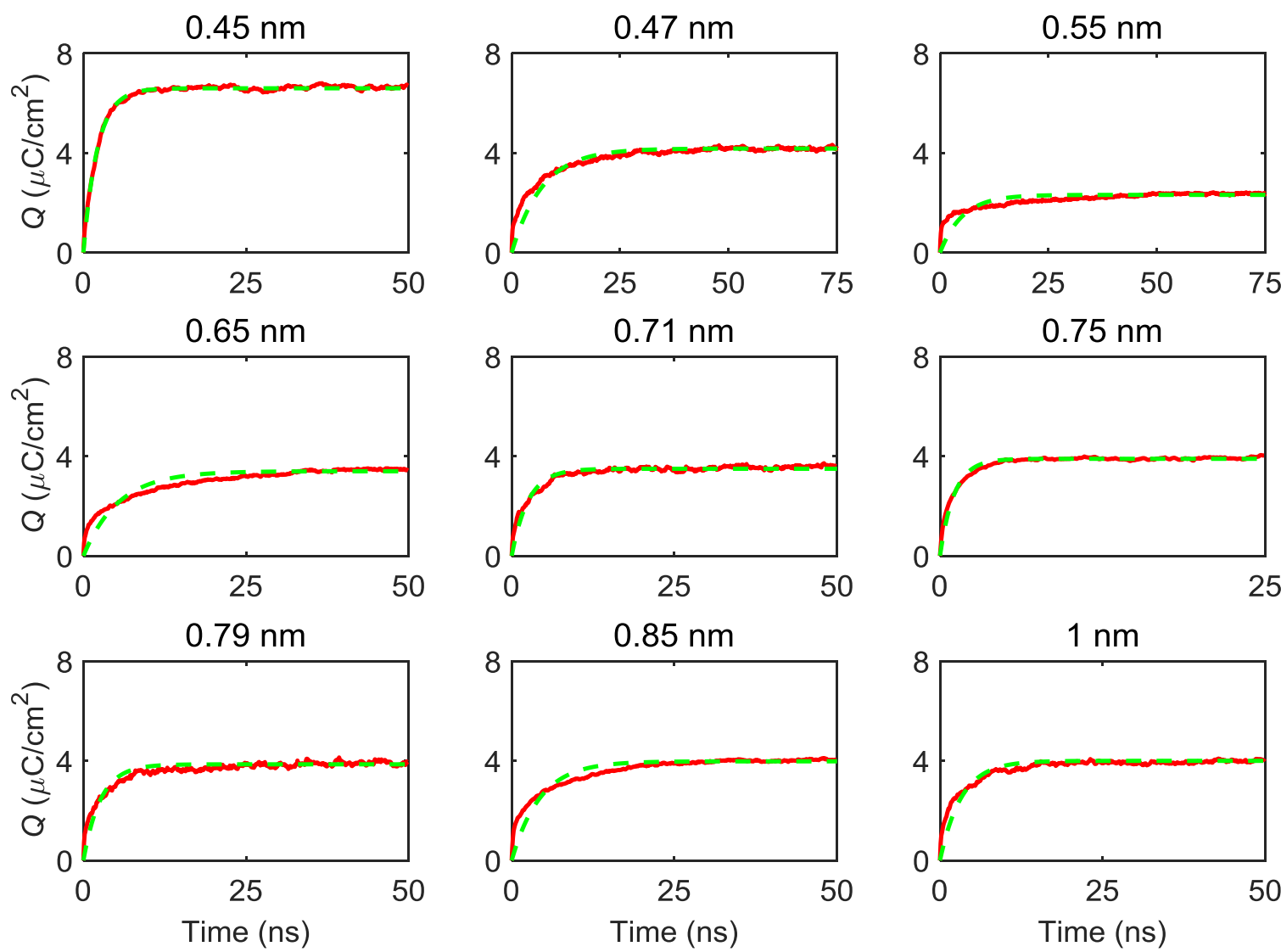

Figure S2 | Total accumulated charge on the electrodes as a function of time for different pore

sizes. Time evolution of charge density, per unit surface area of the pore, after a potential difference of $2 \mathrm{~V}$ was applied between the two electrodes, displayed by the positive electrode, with different pore sizes. In each panel, red solid lines represent the data from molecular dynamics simulations, and green dashed lines represent the curves by fitting molecular dynamics data to the equivalent circuit model. The time length scale was chosen to ensure that the simulation has arrived at the equilibrium. 


\section{Part 3. The capacitive behavior for different pore sizes}

The integral capacitance of the negative and positive electrodes is calculated by $C=Q_{\infty} /\left(\Delta \varphi-\Delta \varphi_{0}\right)$, where $Q_{\infty}$ denotes the charge density on the electrode after reaching an equilibrium, $\Delta \varphi$ represents the potential difference between the electrode (positive or negative electrode) and the bulk electrolyte after applying voltage, and $\Delta \varphi_{0}$ is the potential difference between the electrode and the bulk electrolyte at zero electrode potential. The total integral capacitance is calculated from the potential difference between the two electrodes. The results in Figure S3 show that the electrode capacitance and total capacitance display a similar trend with that in previous experimental and modeling work. ${ }^{1-5}$ The capacitance in this work is close to the previous work where the coarse-grained force field was developed for [EMIM] $\left[\mathrm{BF}_{4}\right]$ employed in our simulation. ${ }^{6}$ Specifically, the capacitance of $[\mathrm{EMIM}]\left[\mathrm{BF}_{4}\right] /$ graphite interface is $4.2 \mu \mathrm{F} / \mathrm{cm}^{2}$ at the positive electrode and $4.9 \mu \mathrm{F} / \mathrm{cm}^{2}$ at the negative electrode. ${ }^{6}$

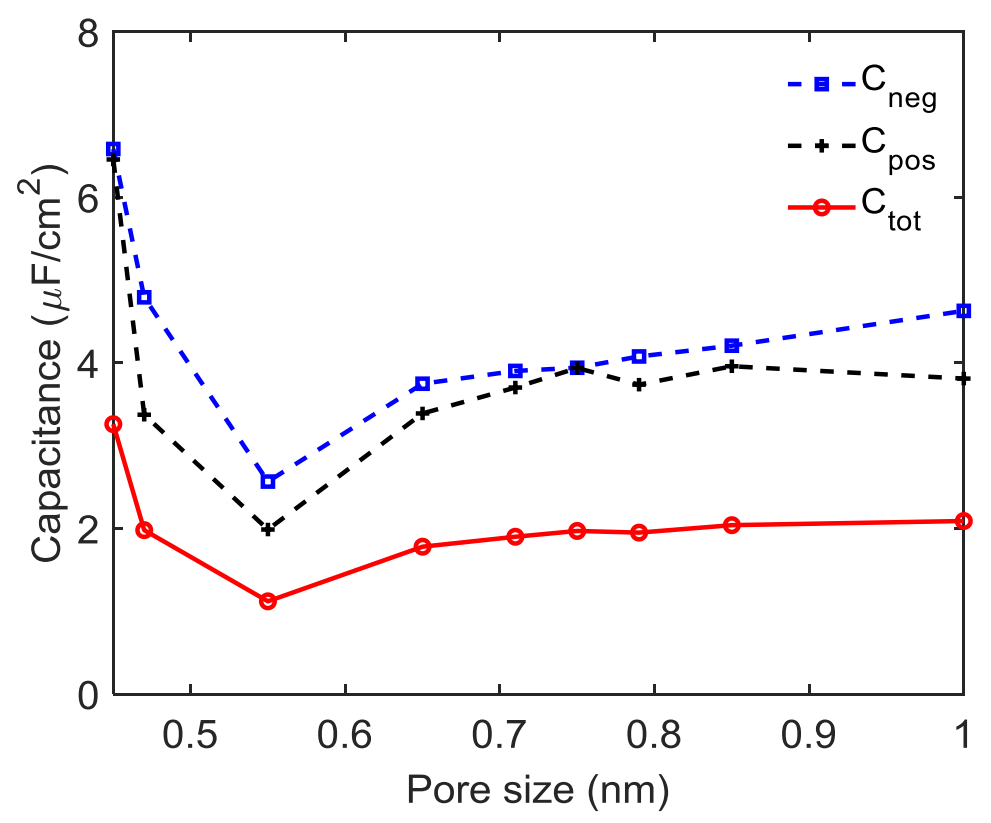

Figure S3 | Specific capacitance as a function of pore size. The red solid line is the total integral capacitance in the simulated system. The blue and black dashed lines represent the integral capacitance under positive and negative polarizations, respectively. 


\section{Part 4. In-pore ion exchange during the charging process}

To describe the role of cations and anions during the charging process, we introduce the net charging mechanism parameter, developed based on the work from Forse et al., ${ }^{7}$ defined as:

$$
\Delta X=X_{\text {pos }}-X_{\text {neg }}
$$

where $X_{\text {pos }}$ and $X_{\text {neg }}$ are the charging mechanism parameters of the positive and negative electrodes, respectively. $\Delta X>0$ indicates anion-dominated charge storage in the system. Specifically, there are three situations for $\Delta X>0$ :

1) $X_{\text {pos }}>0, X_{n e g}>0$. Counterion adsorption is dominant the charging at both positive and negative electrodes, while the counterion (anion) adsorption under the positive polarization is more dominant; thus, anions dominate the charge storage process in the system.

2) $X_{\text {pos }}>0, X_{n e g}<0$. Counterion (anion) adsorption is dominant the charging dynamics under the positive polarization, while the co-ion (anion) desorption is prevailing under the negative polarization; thus, anions are predominant in the system.

3) $X_{\text {pos }}<0, X_{n e g}<0$. Co-ion desorption is predominant under both positive and negative polarizations, while the co-ion desorption (anion) under the negative polarization is more dominant; thus, anions dominate the charge storage process in the system.

For the same reasons, $\Delta X=0$ means that the cation and anion have the equivalent contribution to the charge storage process, and $\Delta X<0$ means that cation is dominant. The conclusions are established for a charge storage system within $+1 \geq X \geq-1$. 

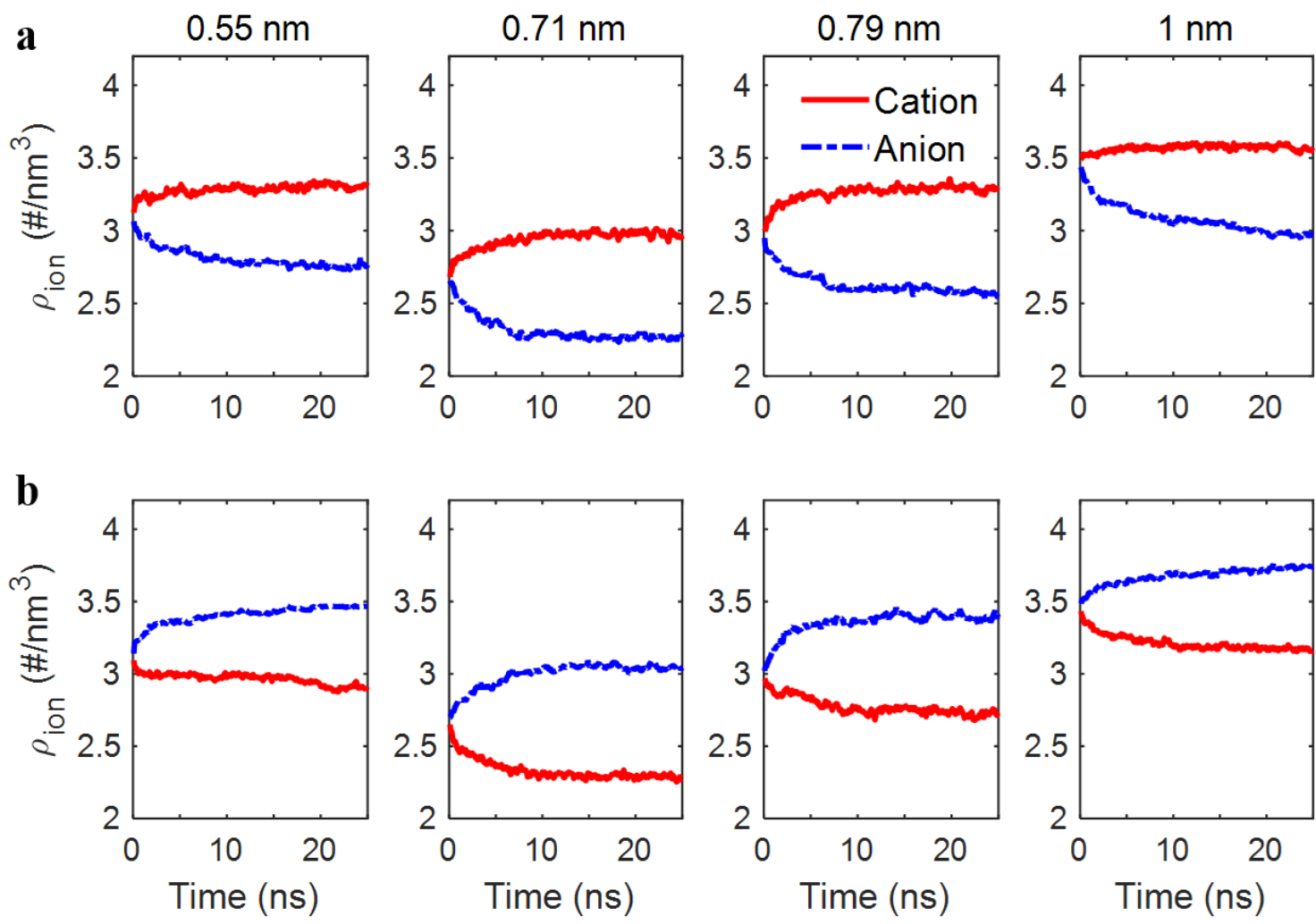

Figure S4 | In-pore ion population changes during the charging process at different pore sizes.

$\mathbf{a}-\mathbf{b}$, Evolution of the average ions number density, $\rho_{\text {ion }}$, inside under negative polarization (a) and under positive polarization (b) during the charging process for pores of different sizes. 


\section{Part 5. In-pore ion distribution}

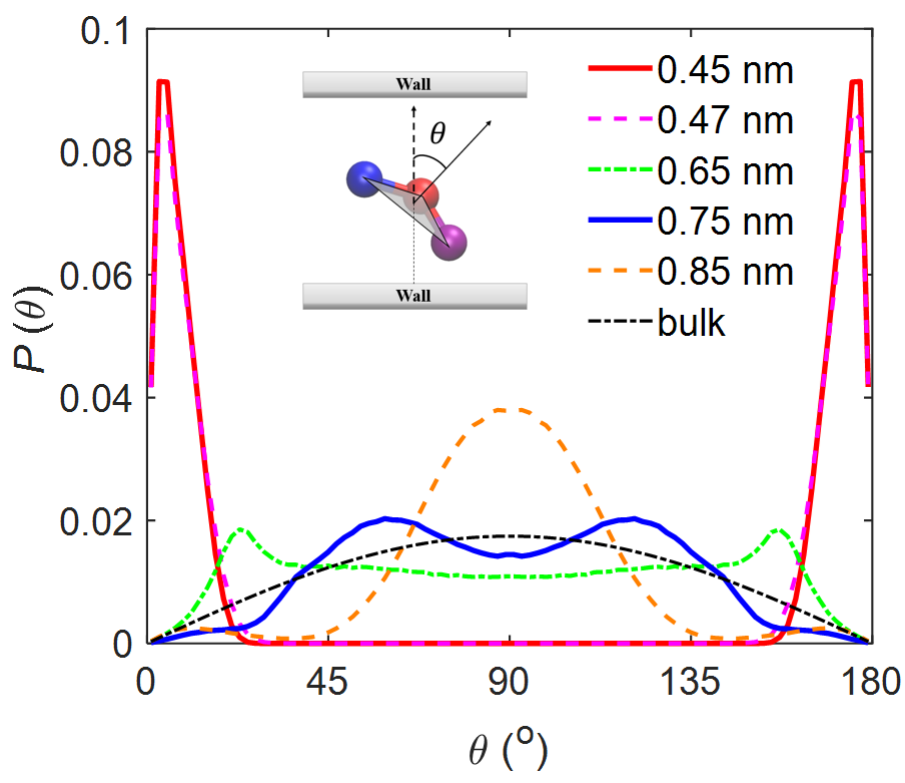

Figure S5 | Orientation distributions inside subnanometer pores. Angle distribution, $P(\theta)$, between the plane of a cation and the pore wall. The inset is a schematic of the cation orientation.
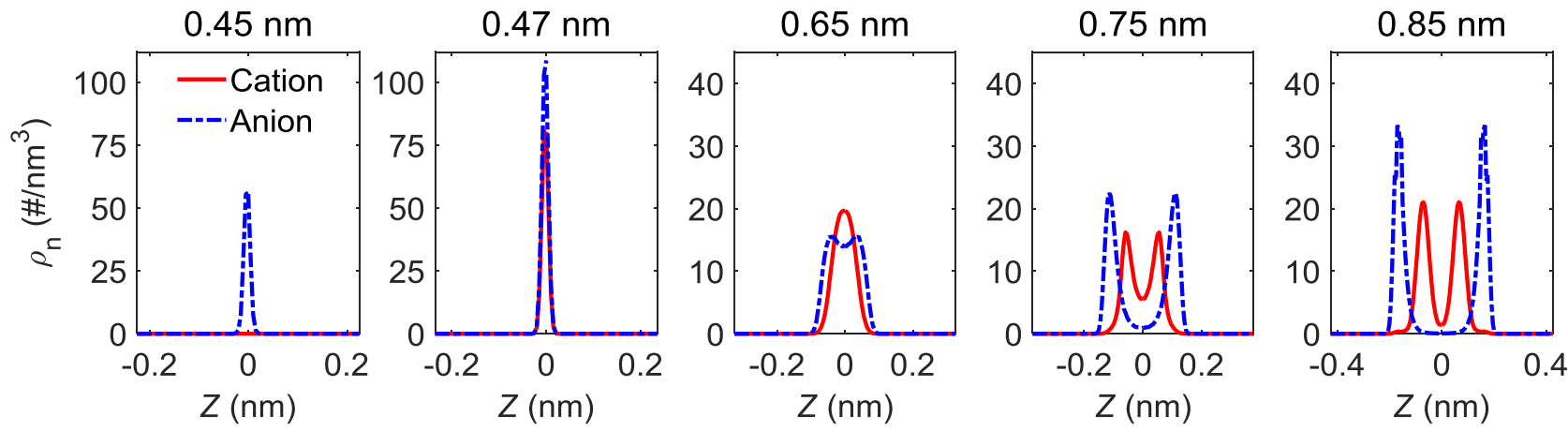

Figure S6 | Number density profiles, $\rho_{n}$, with various pore sizes under positive polarization. The position of ion is based on its mass center, and $Z=0$ represents the position of the central plane between two pore walls. 

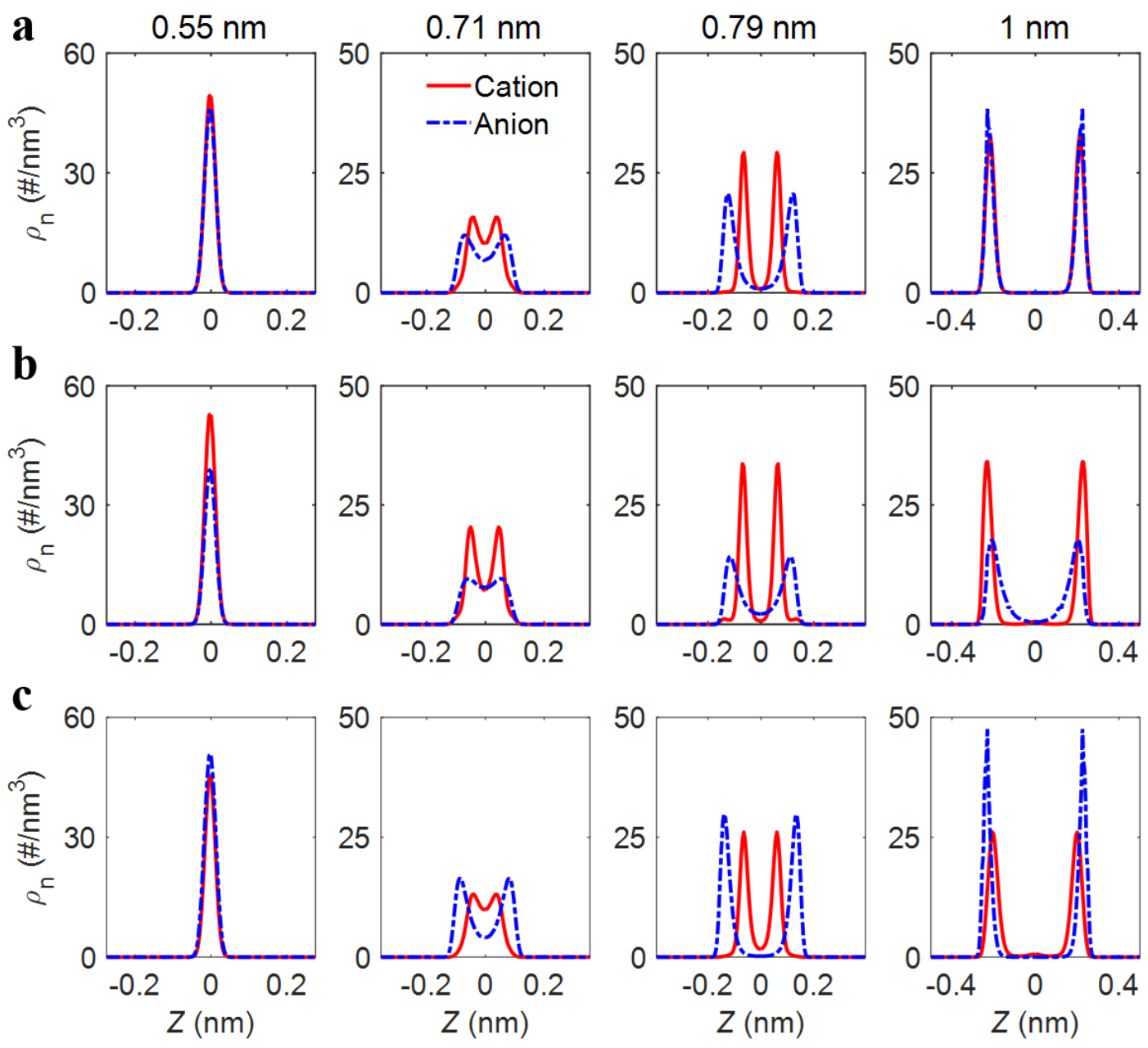

Figure S7 | Number density profiles, $\rho_{n}$, of ions across slit pores with various pore sizes. a-c, The number density of ions inside pores at zero electrode potential (a), under negative polarization (b), and under positive polarization (c). The position of an ion is based on its mass center, and $Z=0$ represents the position of the central plane between two pore walls. 

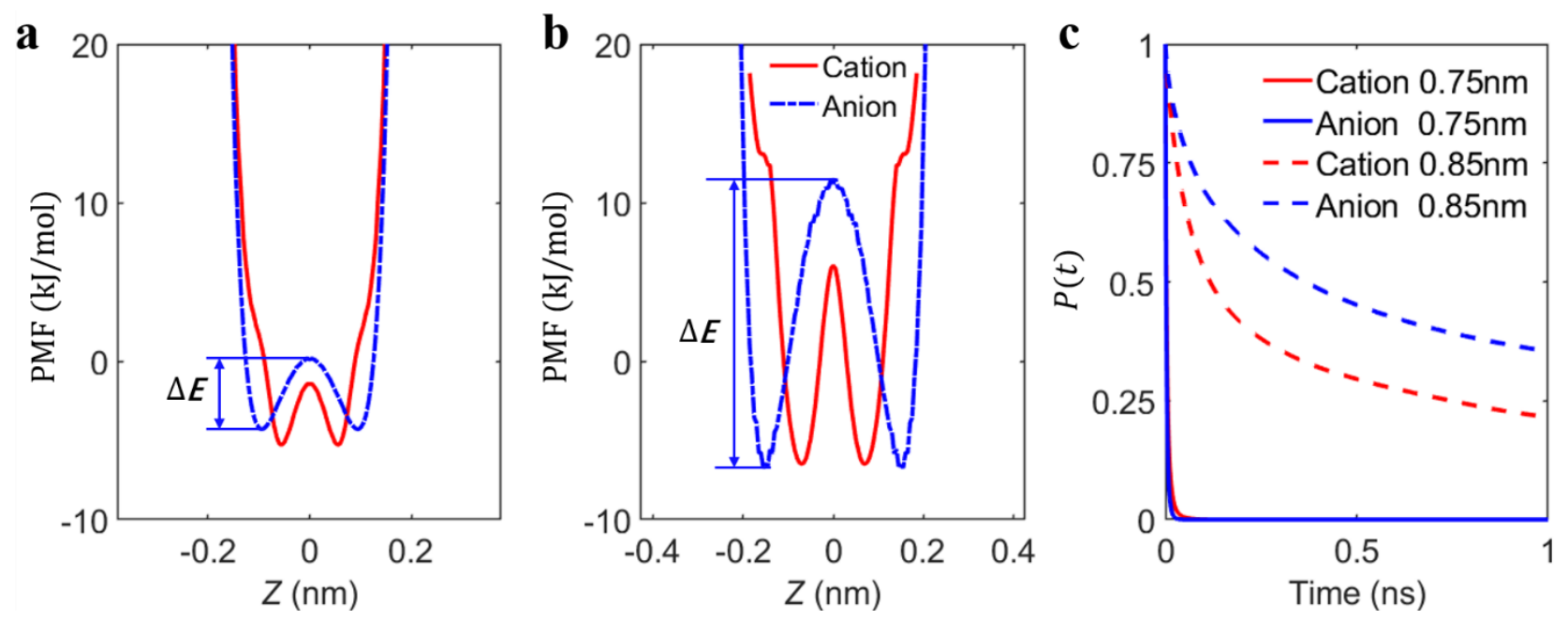

Figure S8 | Ion transport inside the pore at zero electrode potential. a-b, Potential of mean force (PMF) in pores with sizes of $0.75 \mathrm{~nm}$ (a) and $0.85 \mathrm{~nm}(\mathbf{b}) . \Delta E$ is the energy barrier for ions transporting from one layer to the other. c, Survival probability of ions in an ion layer, $P(t)=$ $\frac{N\left(t_{0}, t_{0}+t\right)}{N\left(t_{0}\right)}$, where $N\left(t_{0}\right)$ is the number of ions in one layer at $t_{0}$, and $N\left(t_{0}, t_{0}+t\right)$ is the number of ions remaining in such a layer at $t_{0}+t . Z=0$ represents the position of the central plane between two pore walls.
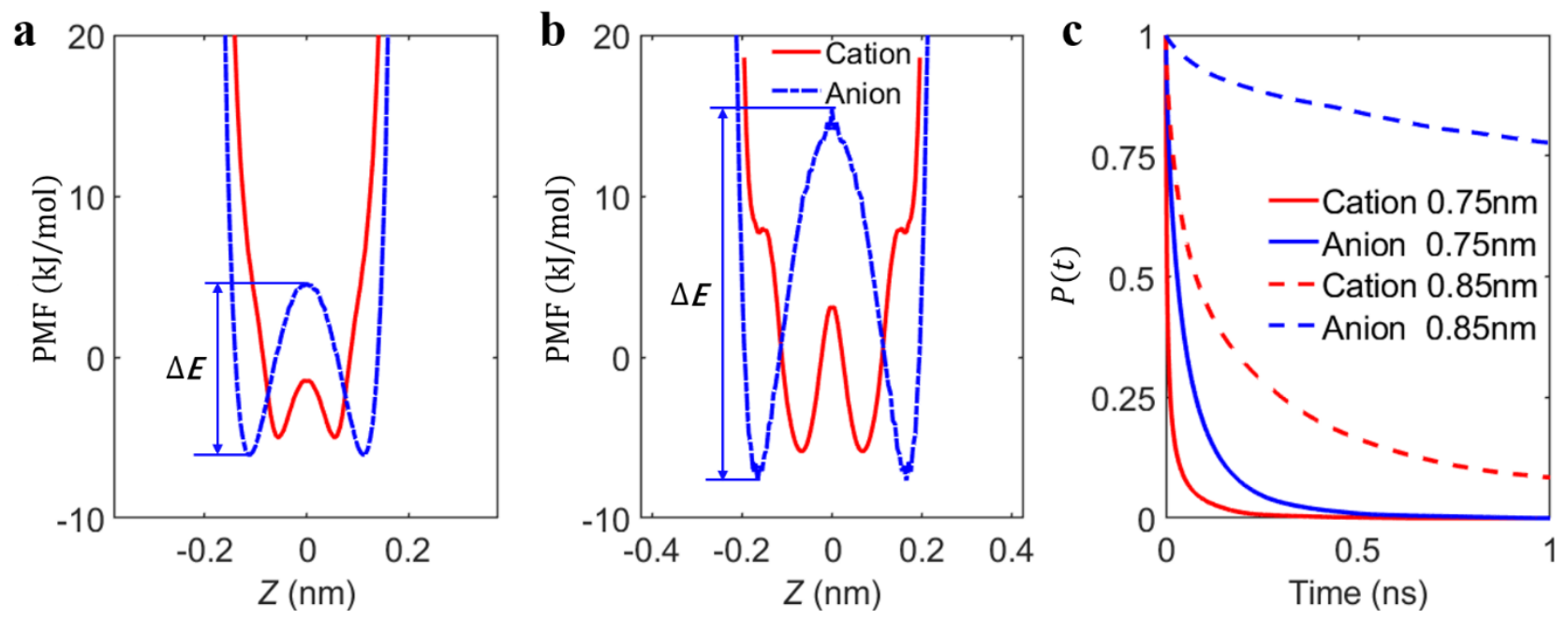

Figure S9 | Ion transport inside the pore under positive polarization. a-b, Potential of mean force (PMF) in pores with sizes of $0.75 \mathrm{~nm}(\mathbf{a})$ and $0.85 \mathrm{~nm}(\mathbf{b}) . \Delta E$ is the energy barrier for ions transporting from one layer to the other. c, Survival probability of ions in an ion layer. $Z=0$ represents the position of the central plane between two pore walls. 
Table S1 | The energy barrier and the residence time.

\begin{tabular}{cccccccc}
\hline \multirow{2}{*}{$\begin{array}{c}\text { Pore } \\
\text { size } \\
(\mathbf{n m})\end{array}$} & $\begin{array}{c}\text { Ion } \\
\text { type }\end{array}$ & $\begin{array}{c}\text { Zero } \\
\text { electrode } \\
\text { potential }\end{array}$ & $\begin{array}{c}\text { Negative } \\
\text { polarization }\end{array}$ & $\begin{array}{c}\text { Positive } \\
\text { polarization }\end{array}$ & $\begin{array}{c}\text { Zero } \\
\text { electrode } \\
\text { potential }\end{array}$ & $\begin{array}{c}\text { Negative } \\
\text { polarization }\end{array}$ & $\begin{array}{c}\text { Positive } \\
\text { polarization }\end{array}$ \\
\hline \multirow{2}{*}{0.75} & Cation & 3.82 & 9.45 & 3.19 & 5.48 & 26.81 & 17.11 \\
\cline { 2 - 8 } & Anion & 4.42 & 2.51 & 10.55 & 3.64 & 4.39 & 64.87 \\
\hline \multirow{2}{*}{0.85} & Cation & 12.43 & 11.96 & 9.52 & 778.22 & 368.08 & 425.21 \\
\cline { 2 - 7 } & Anion & 18.26 & 6.02 & 22.86 & 1518.08 & 130.24 & - \\
\hline
\end{tabular}

Note: The residence time was calculated by $\tau_{r}=\int_{0}^{\infty} P(t) d t$. 
a

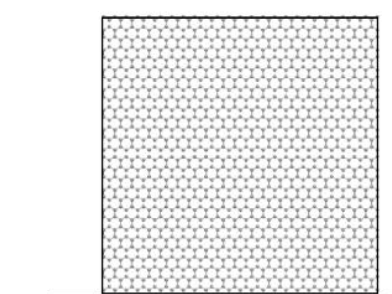

b

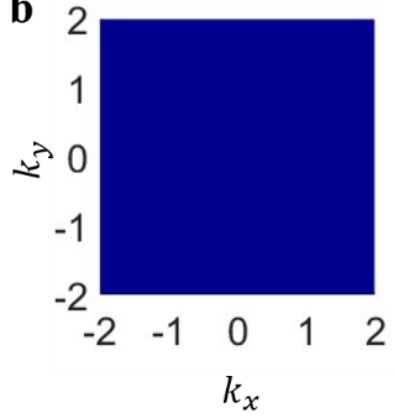

$0.47 \mathrm{~nm}$
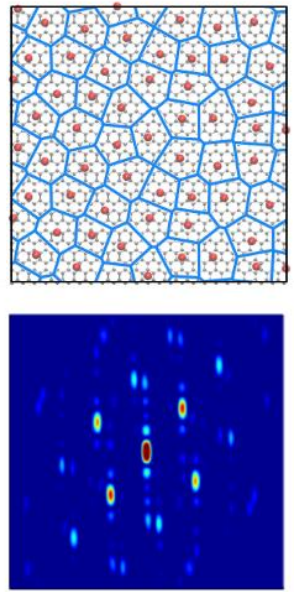

$2-2-1$

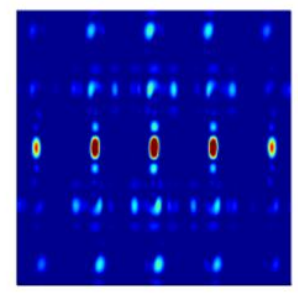

$0.65 \mathrm{~nm}$
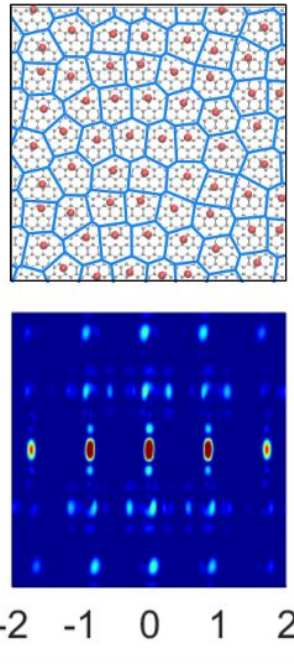

$0.75 \mathrm{~nm}$
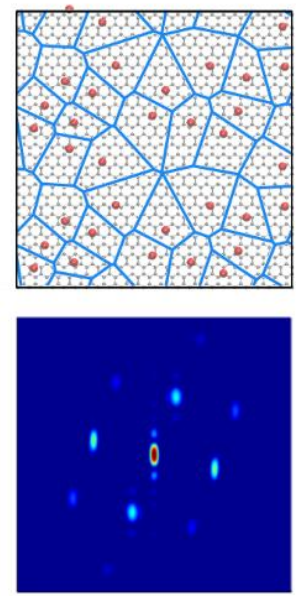

$2-2-1$
$0.85 \mathrm{~nm}$
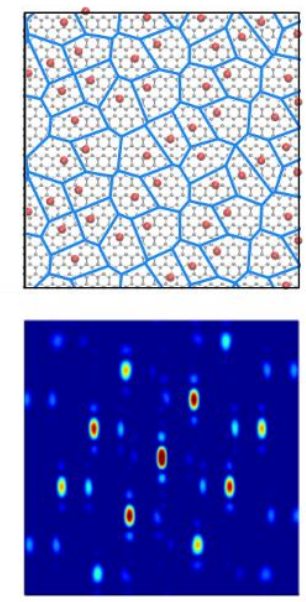

$\begin{array}{llllll}2 & -2 & -1 & 0 & 1 & 2\end{array}$

Figure S10 | In-plane cation structure inside pores with various pore size at zero electrode

potential. a, Top-view snapshots, and Voronoi tessellations, composed by a set of vertical bisectors connecting two adjacent cations in the ion layer, inside the pore, the green spheres represent cations, and the hexagonal network in the background represents wall atoms, respectively. The blue grids are Voronoi tessellations. b, Structure factor of cations inside the pore. The structure factor is calculated by $S(\boldsymbol{k})=<\frac{1}{N} \sum_{m=1}^{N} \sum_{n=1, m \neq n}^{N} e^{-i \boldsymbol{k} \cdot \boldsymbol{r}_{m, n}}>$, where $<>$ denotes the ensemble average, $\boldsymbol{k}$ is the scattering vector parallel to the electrode wall, $N$ is the number of cations in the first adsorption layer, and $\boldsymbol{r}_{m, n}$ is the vector pointing from ion $m$ to ion $n$. 
$\mathbf{a}$

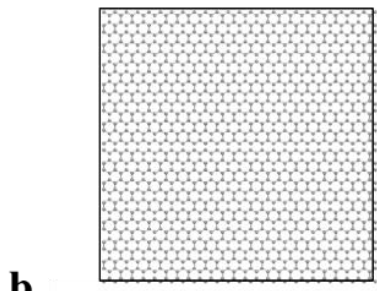

b

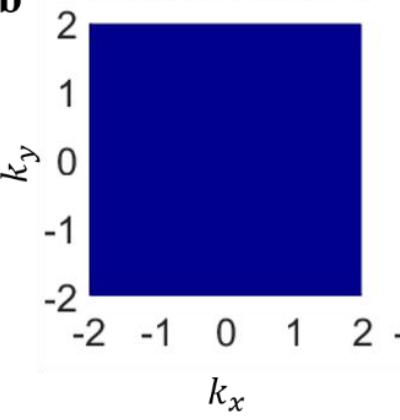

$0.47 \mathrm{~nm}$
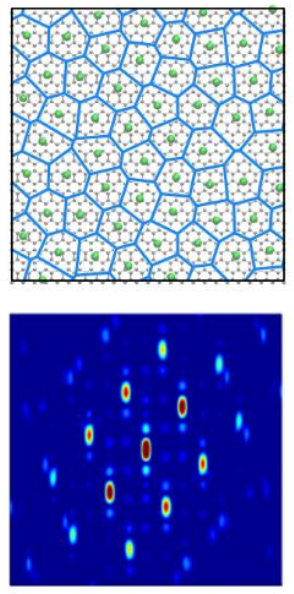

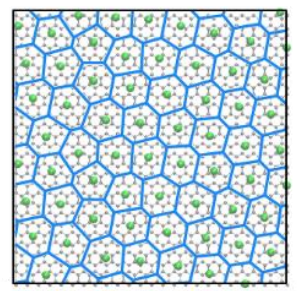

$0.75 \mathrm{~nm}$
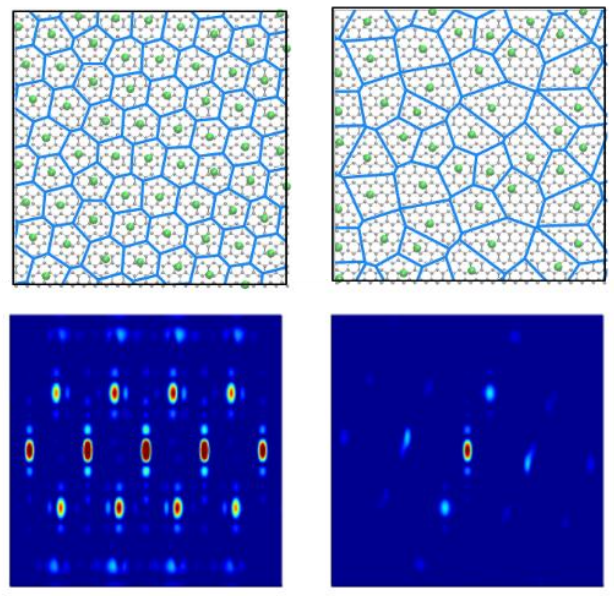

$\begin{array}{llllllllllllllll}2 & -2 & -1 & 0 & 1 & 2 & -2 & -1 & 0 & 1 & 2 & -2 & -1 & 0 & 1 & 2\end{array}$ $k_{x}$

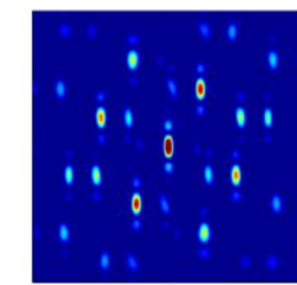

$0.85 \mathrm{~nm}$

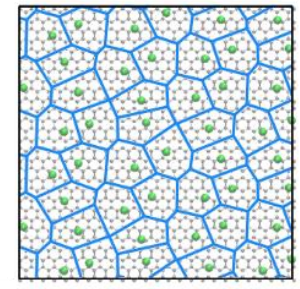

$k_{x}$

$k_{x}$

Figure S11 | In-plane anion structure inside pores with various pore size at zero electrode

potential. a, Top-view snapshots, and Voronoi tessellations, composed by a set of vertical bisectors connecting two adjacent anions in the ion layer, inside the pores, the green spheres represent anion, and the hexagonal network in the background represents wall atoms, respectively. The blue grids are Voronoi tessellations. b, Structure factor of anion layer inside the pores. The blue grids are Voronoi tessellations. The structure factor is calculated by $S(\boldsymbol{k})=<\frac{1}{N} \sum_{m=1}^{N} \sum_{n=1, m \neq n}^{N} e^{-i \boldsymbol{k} \cdot \boldsymbol{r}_{m, n}}>$, where $<>$ denotes the ensemble average, $\boldsymbol{k}$ is the scattering vector parallel to the electrode wall, $N$ is the number of anions in the first adsorption layer, and $\boldsymbol{r}_{m, n}$ is the vector pointing from ion $m$ to ion $n$. 

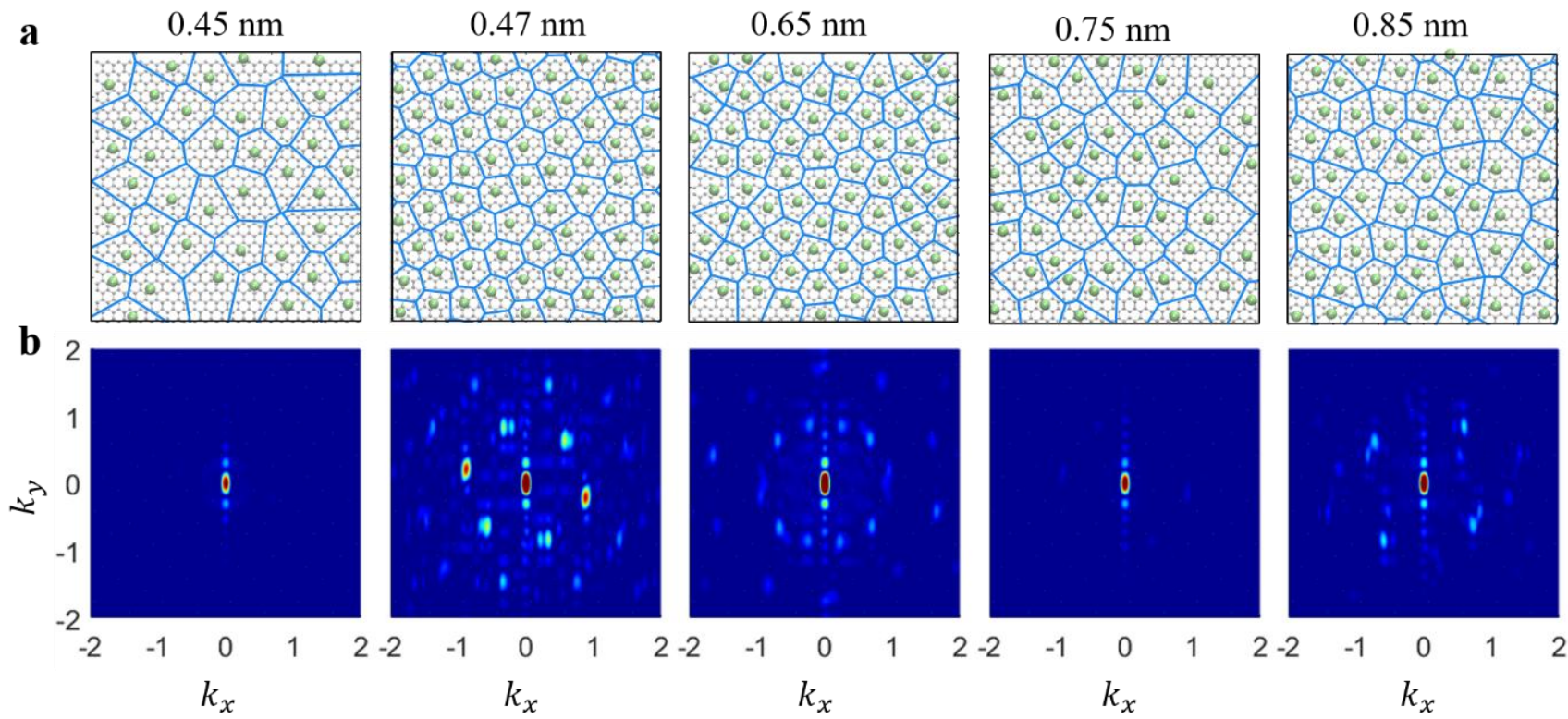

Figure S12 | In-plane anion structure inside pores with various pore sizes under positive

polarization. a, Top-view snapshots and Voronoi tessellations, composed by a set of vertical bisectors connecting two adjacent anions in the anion layer, inside the positive electrode, the green spheres represent anions, and the hexagonal network in the background represents wall atoms, respectively. The blue grids are Voronoi tessellations. b, Structure factor of anion layer inside the positive electrodes. The blue grids are Voronoi tessellations. The structure factor is calculated by $S(\boldsymbol{k})=<\frac{1}{N} \sum_{m=1}^{N} \sum_{n=1, m \neq n}^{N} e^{-i \boldsymbol{k} \cdot \boldsymbol{r}_{m, n}}>$, where $<>$ denotes the ensemble average, $\boldsymbol{k}$ is the scattering vector parallel to the electrode wall, $N$ is the number of anions in the first adsorption layer, and $\boldsymbol{r}_{m, n}$ is the vector pointing from ion $m$ to ion $n$. 
Part 6. Ion diffusion in pores of different size

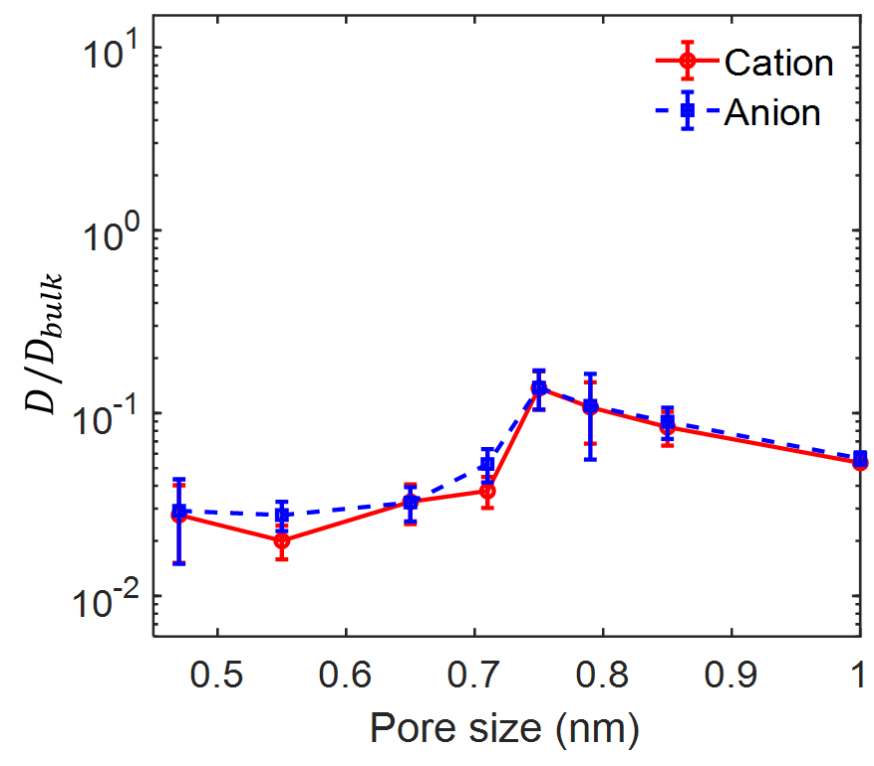

Figure S13 | Diffusion coefficient at zero electrode potential as a function of pore size. The ion diffusion coefficient is expressed in terms of the diffusion coefficient of ions in bulk RTIL system $\left(D_{\text {bulk }}\right)$. The diffusion coefficients of cation and anion in the bulk system at $400 \mathrm{~K}$ are $53.8 \times 10^{-11} \mathrm{~m}^{2} / \mathrm{s}$ and $38 \times 10^{-11} \mathrm{~m}^{2} / \mathrm{s}$, respectively. 
Part 7. Charging dynamics of nanopore larger than $1 \mathbf{~ n m}$

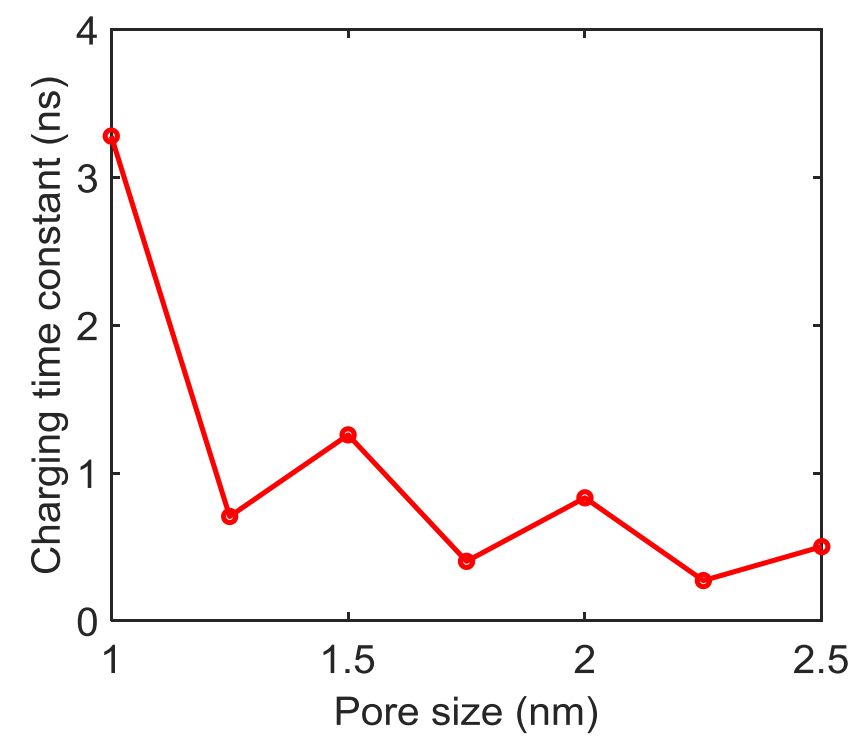

Figure S14 | Charging time constant as a function of pore size ranging from $1.0 \mathrm{~nm}$ to $2.5 \mathrm{~nm}$. The charging time constant is calculated by fitting the MD-obtained charging curve to equivalent circuit model (see Eq. 1 in main text). 


\section{References}

1. Feng, G.; Cummings, P. T., Supercapacitor Capacitance Exhibits Oscillatory Behavior as a Function of Nanopore Size. J. Phys. Chem. Lett. 2011, 2, 2859-2864.

2. Wu, P.; Huang, J.; Meunier, V.; Sumpter, B. G.; Qiao, R., Complex Capacitance Scaling in Ionic Liquids-Filled Nanopores. ACS Nano 2011, 5, 9044-9051.

3. Jiang, D.; Jin, Z.; Wu, J., Oscillation of Capacitance inside Nanopores. Nano Lett. 2011, 11, 5373-5377.

4. Chmiola, J.; Yushin, G.; Gogotsi, Y.; Portet, C.; Simon, P.; Taberna, P.-L., Anomalous Increase in Carbon Capacitance at Pore Sizes Less Than 1 Nanometer. Science 2006, 313, 1760-1763.

5. Largeot, C.; Portet, C.; Chmiola, J.; Taberna, P.-L.; Gogotsi, Y.; Simon, P., Relation between the Ion Size and Pore Size for an Electric Double-Layer Capacitor. J. Am. Chem. Soc. 2008, 130, 2730-2731.

6. Merlet, C.; Salanne, M.; Rotenberg, B., New Coarse-Grained Models of Imidazolium Ionic Liquids for Bulk and Interfacial Molecular Simulations. J. Phys. Chem. C 2012, 116, 7687-7693.

7. Forse, A. C.; Merlet, C.; Griffin, J. M.; Grey, C. P., New Perspectives on the Charging Mechanisms of Supercapacitors. J. Am. Chem. Soc. 2016, 138, 5731-5744. 